\title{
CAPITAL HUMANO, EMPREENDEDORISMO E DESENVOLVIMENTO: EVIDÊNCIAS EMPÍRICAS NOS MUNICÍPIOS DO CEARÁ
}

\section{RAIMUNDO EDUARDO SILVEIRA FONTENELE}

Doutor em Economia pelo Centre D’Economie de L'Université Paris Nord da Universidade de Paris XIII. Professor do Programa de Pós-Graduação em Administração da Universidade de Fortaleza (Unifor) Avenida Washington Soares, 1.321, bloco Q, sala 3, Edson Queiroz, Fortaleza - CE - Brasil - CEP 6081 1-905

\section{HEBER JOSÉ DE MOURA}

Doutor em Administração pelo Departamento de Administração da Fundação Getulio Vargas de São Paulo (FGV-SP). Professor do Programa de Pós-Graduação em Administração da Universidade de Fortaleza (Unifor). Avenida Washington Soares, 1.321, bloco Q, sala 3, Edson Queiroz, Fortaleza - CE - Brasil - CEP 6081 1-905 E-mail: heberm@unifor.br

\section{AURIO LUCIO LEOCADIO}

Doutor em Administração pela Faculdade de Economia, Administração e Contabilidade da Universidade de São Paulo (USP).

Professor do Setor de Ciências Sociais e Aplicadas da Universidade Federal do Paraná (UFPR). Avenida Prefeito Lothário Meissner, 632, Jardim Botânico, Curitiba - PR - Brasil - CEP 80210-170 E-mail: alleocadio@uol.com.br 


\section{RESUMO}

A contribuição do capital humano para o processo de desenvolvimento econômico das nações já foi objeto de vários estudos desde a década de i960. Entretanto, a atualização do tema começou na segunda metade dos anos I990, com a necessidade de analisar o papel do empreendedorismo nesse processo. Este artigo estuda a influência do capital humano e do empreendedorismo no processo de desenvolvimento econômico. Para o estudo, reuniu-se uma base de dados de indicadores sociais, demográficos, econômicos e de infraestrutura de todos os I84 municípios cearenses, a partir de dados secundários fornecidos pelo Instituto de Pesquisa e Estratégia Econômica do Ceará (Ipece) e pelo Instituto Brasileiro de Geografia e Estatística (IBGE), e realizaram-se três técnicas de análise para confirmar as hipóteses formuladas com base na literatura publicada: análise fatorial, análise de regressão e modelo de equações estruturais. Os resultados das análises reforçam a hipótese de que a capacidade empreendedora nesse Estado tem consistido apenas em mais uma alternativa de trabalho e não como uma contribuição para o desenvolvimento e crescimento econômicos. Tendo por objetivo a integração dos construtos do estudo e análise de suas relações, utilizou-se a modelagem de equações estruturais. Os dados obtidos no modelo estão coerentes com os resultados da análise de regressão múltipla realizada, confirmando a existência de relação do capital humano com o desenvolvimento. Apesar das limitações da medida do empreendedorismo pela atividade dos trabalhadores por conta própria, os resultados do estudo confirmam conclusões obtidas em outros trabalhos: o empreendedorismo pode ser diferente, dependendo do estágio de desenvolvimento do país (AGHION; HOWITT, 2005), e o impacto do empreendedorismo sobre as taxas de crescimento econômico é negativo (BARROS; PEREIRA, 2008). Como pesquisa futura, sugere-se a construção de um modelo que contemple outras variáveis explicativas, possibilitando ampliar o conhecimento do papel do empreendedorismo e do capital humano no desenvolvimento. Outra possibilidade seria a extensão da pesquisa fazendo comparações com outros Estados da Federação e/ou entre países, bem como replicar este estudo longitudinalmente em outros anos. 


\section{PALAVRAS-CHAVE}

Capital humano; Empreendedorismo; Crescimento econômico; Desenvolvimento; Ceará.

\section{INTRODUÇ $\tilde{A} O$}

O papel do capital humano tem sido uma preocupação de um número expressivo de pesquisadores, notadamente os economistas ligados ao crescimento econômico.

A maior parte da literatura sobre o assunto, entretanto, dominada pelo paradigma neoclássico, não integrou o papel do empreendedor nessas análises. Essa ausência do empreendedor se deve especialmente às dificuldades teóricas, o que acarreta graves lacunas na compreensão dos mecanismos básicos de funcionamento da economia.

Apesar de o espírito empreendedor ter sido excluído na teoria de crescimento econômico, muitos economistas postulam que a capacidade empreendedora é vital para o progresso econômico, especialmente com as contribuições de Schumpeter (I96I) e Kirzner (I973).

Schumpeter (I96I) expandiu o conceito de empreendedorismo com a introdução da noção de "destruição criativa", na qual demonstrava que o empreendedor, ao incorporar inovações e novas tecnologias, contribuía para a substituição de produtos e processos ultrapassados. Assim, o empreendedor assumia não apenas um papel central no avanço e desenvolvimento da economia e da sociedade, como protagonizava também um papel fundamental na evolução da vida empresarial e na substituição das empresas estabelecidas por novas organizações mais capazes de aproveitar as inovações.

A limitação dos modelos neoclássicos não se restringe apenas à exclusão do empreendedorismo em suas análises. Com base na revisão de literatura, percebe-se que, somente depois da década de ig80, sobretudo com os trabalhos de Paul Romer e Robert Lucas, as teorias de crescimento econômico passaram a inserir nos modelos tradicionais variáveis não necessariamente econômicas, como é o caso da formação do capital humano como um dos principais determinantes de crescimento de longo prazo (SALA-I-MARTIN, I995; BLEANEY; NISHIYAMA, 2002).

Essa evolução na teoria do crescimento econômico, com a inserção do capital humano, da tecnologia e da inovação na função de produção, possibilitou um melhor arcabouço teórico e científico, visando compreender melhor a evolução 
do Produto Interno Bruto (PIB) dos países ao longo do tempo, visto que acrescentaram ao modelo original variáveis como capital humano e tecnologia/inovação.

Nos últimos anos, destacaram-se as pesquisas que enfatizam o papel do capital humano em favorecer maiores níveis de produtividade. Do mesmo modo, outra variável que vem sendo muito investigada no período recente refere-se ao poder do empreendedorismo em promover esse crescimento, pela percepção de que os empreendedores possuem um importante papel de agente inovador na economia, com transformações decorrentes da introdução de novos produtos no mercado, pelas mudanças tecnológicas e por mudanças nos processos produtivos (ACS; AUDRETSCH, I990).

O presente estudo pretende aliar essas duas teorias, tendo como principal objetivo identificar a influência do capital humano e do empreendedorismo para o crescimento e o desenvolvimento econômico dos I84 municípios do Estado do Ceará. De modo a prosseguir com esse objetivo geral, houve a necessidade de delimitar objetivos mais específicos. Assim, foram definidos os seguintes objetivos: I. identificar os fatores que determinam o capital humano; 2. analisar a relação do empreendedorismo com o crescimento e o desenvolvimento econômico e 3. analisar a relação do capital humano com o crescimento e o desenvolvimento econômico dos municípios do Estado do Ceará.

A relevância deste estudo está fundamentada na possibilidade de iniciar um processo de entendimento sobre o impacto do processo de formação de capital humano e capacidade empreendedora no crescimento econômico desses municípios, o que contribuirá para a elaboração de políticas locais direcionadas.

Para alcançar esse objetivo, serão utilizados procedimentos existentes de análise multivariada, mais especificamente o método de análise fatorial com decomposição em componentes principais, o método dos mínimos quadrados ordinários (MQO) e uso de modelagem de equações estruturais.

\section{- papel do capital humano no CRESCIMENTO ECONÔMICO}

A literatura que estuda o crescimento econômico é vasta e rica em modelos que tentam explicar as variáveis que causam e geram o crescimento da renda nacional. Deve-se aos trabalhos de Solow, na década de I950, uma mudança na compreensão contemporânea do crescimento econômico, a partir da incorporação da acumulação de capital humano como tão importante como a acumulação do capital. Desde então, ocorreram vários aprofundamentos, como o modelo exposto por Mankiw, Romer e Weil (I992) nos anos I990 em que inserem a variável capital humano, introduzindo a variável progresso técnico (KROTH; DIAS, 2006). 
Segundo Dias e Dias (I999), a importância do capital humano para o crescimento do produto se deve ao fato de que os indivíduos com maiores habilidades e conhecimento tornam-se mais produtivos, contribuindo para gerar um maior nível de produção, além de possibilitar a inovação das técnicas de produção. Essa linha teórica, denominada teoria do capital humano, foi proposta por Theodore Schultz em ig60 com o intuito de questionar os estudos tradicionais da economia neoclássica que evidenciavam empiricamente que somente o acúmulo de fatores de produção fosse o responsável por gerar crescimento econômico (FRANK, 2008).

Para Marteleto e Silva (2004), o capital humano engloba as habilidades e os conhecimentos dos indivíduos que, em conjunto com outras características pessoais e o esforço despendido, aumentam as possibilidades de produção e de bem-estar pessoal, social e econômico.

Os estudos sobre capital humano têm início na década de i960, com destaque para as pesquisas em nível microeconômico, que buscavam mensurar os retornos com investimentos em educação (KROTH; DIAS, 2006).

Foi, entretanto, Schultz (I962, p. I, tradução nossa) que estabeleceu as relações macroeconômicas entre capital humano e crescimento econômico:

A hipótese aqui apresentada é a de que a adição do capital humano mostrará que a relação entre capital e renda não decresce. A produção de bens físicos como “instalações, equipamentos e estoques" tem declinado em relação à renda. Contudo, o capital humano tem crescido relativamente à renda. Se a relação entre os capitais e a renda permanecer constante, então um eventual crescimento econômico, embora confuso, é originário do crescimento do estoque de capital humano.

Assim, para Schultz (I962), a inclusão da acumulação de capital humano é um elemento-chave na compreensão do crescimento econômico, no longo prazo, pois ele é a principal fonte desse processo. Posteriormente, os trabalhos de Lucas Jr. (I988) corroboraram o tema, demonstrando sua importância na geração de externalidades positivas sobre a produtividade ao longo do tempo, favorecendo assim o crescimento econômico sustentado.

Muito embora esses autores tenham desenvolvido vários trabalhos que apontam o capital humano como um fator determinante para o crescimento/ desenvolvimento de um país, ainda existem muitas controvérsias sobre a importância desse fator no crescimento econômico/nível de renda. Alguns estudos, como Romer (I990), Benhabib e Spiegel (I994) e Pritchett (I996), encontram resultados que colocam em dúvida a suposição de que o capital humano é um 
importante fator na determinação da renda de forma direta. Por forma direta, consideram-se os efeitos do capital humano sobre a renda por meio da melhora marginal da produtividade do trabalho, mantendo todos os outros fatores constantes (capital e tecnologia).

Existem, porém, muitos outros estudos que dão suporte a essa hipótese, com destaque para Barro (I99I), Mankiw, Romer e Weil (I992) e Barro e Lee (200I), e a maior parte das evidências é proveniente de estudos microeconômicos (DOWRICK, 2003).

Em relação aos trabalhos empíricos desenvolvidos para o Ceará, podem-se destacar os trabalhos de Oliveira Silva (2006), Oliveira (2006), Barreto e Almeida (2009) e Lima et al. (2009). As pesquisas de Oliveira Silva (2006) demonstram evidências de que o capital humano possui retorno superior ao capital físico para promover o crescimento econômico do Estado do Ceará.

Dentro da mesma linha de pensamento, Oliveira (2006) calcula, utilizando um modelo econométrico espacial com dados em painel, as contribuições do crescimento econômico e da redução da desigualdade de renda na redução da pobreza dos municípios da região. Os resultados obtidos mostram que poucos municípios reduziram a pobreza com a redução na desigualdade de renda e que o crescimento econômico foi o responsável pela redução da pobreza na maioria dos municípios em que houve redução desta.

O trabalho de Barreto e Almeida (2009), ao estudar o papel do capital humano para o crescimento econômico e a convergência de renda dos municípios do Estado do Ceará no período de 1996 a 2003, fez uso de um modelo econométrico e mostrou que o crescimento do PIB per capita foi afetado negativamente pela variável densidade demográfica e positivamente pelas variáveis capital humano, infraestrutura, capital social, mercado regional e externalidades espaciais.

As pesquisas de Lima et al. (2009) identificaram as variáveis que contribuem de forma significativa para um maior nível de capital humano nos municípios cearenses. A primeira parte da pesquisa consistiu na estimativa do Índice de Capital Humano (ICH) para cada um dos municípios do Ceará, sendo criados dois grupos: o primeiro composto pelos 69 municípios com $\mathrm{ICH} \leq 0,5$ e o segundo com os II5 municípios com ICH >0,5. Em seguida, utilizou-se a técnica de análise discriminante para identificar as variáveis que explicam as diferenças entre os dois grupos. Entre as variáveis significantes capazes de explicar as diferenças entre os dois grupos, destacam-se aquelas ligadas à quantificação dos professores mostrando a necessidade de programas contínuos de capacitação desses profissionais na formação do estoque de capital humano dos municípios. 


\section{O CAPITAL hUMANO E EMPREENDEDORISIMO}

A teoria do capital humano postula que o conhecimento proporciona aos indivíduos um aumento de suas competências e habilidades cognitivas, o que pode resultar em mais produtiva e eficiente atividade potencial (SCHULTZ, I962; BECKER, I964; MINCER, I974). Nesse contexto, diante de um cenário de oportunidades rentáveis para novas atividades econômicas, os indivíduos com mais capital humano são os que melhor percebem a exploração de oportunidades de sucesso.

A educação formal é um componente do capital humano que pode contribuir para o acúmulo de conhecimento explícito e que proporciona habilidades úteis para os empresários. O capital humano não é apenas o resultado da educação formal, mas inclui experiências e práticas de aprendizagem que ocorrem no ambiente empresarial, bem como a educação não formal, como cursos de formação específica que não fazem parte das tradicionais estruturas formais de educação. Assim, a experiência no mercado de trabalho e a formação profissionalizante orientada são teoricamente benéficas para aumento do capital humano (BECKER, I964).

Muito embora haja predomínio das pesquisas que identificam uma forte correlação entre capital humano e empreendedorismo, a revisão de literatura constatou que esses resultados não são consensuais. Davidsson e Honig (2003), por exemplo, sugerem que a associação entre o capital humano e o desempenho empresarial pode ser confundida com uma série de outros fatores, tais como a persistência e a experiência profissional.

Segundo Bartlett e Ghoshal (2002), para desenvolver o capital humano, um dos objetivos fundamentais é a disseminação dos conhecimentos. Por definição, empreendedores são indivíduos e não organizações. Portanto, os aspectos culturais relacionados à sua formação ou pelo contexto em que vivem influenciam diferentemente nas nações (LUNDSTRÖM; STEVENSON, 200I). Segundo os autores, em países que possuem uma percepção mais favorável a fatores como risco, competição e individualismo, como os Estados Unidos e alguns países da Europa ocidental, são identificados níveis mais elevados de empreendedorismo do que em países onde essas características não são tão acentuadas, como o Japão.

Muito embora o Brasil seja reconhecido como um dos países mais empreendedores do mundo na atualidade (MINNITI; BYGRAVE; AUTIO, 2006), ainda existem barreiras culturais que podem evidenciar no nível de interesse das pessoas quanto à criação e manutenção de um novo negócio. Apesar de os dados mais recentes do Global Entrepreneurship Monitor (GEM) indicarem uma taxa empreendedora total mais relacionada ao empreendedorismo de oportunidade, culturalmente o brasileiro está ainda bastante arraigado ao conceito de emprego 
e salário, e o sistema de ensino no país vem tradicionalmente preparando o estudante para ser empregado (MAMEDE, 2005).

Assim, muito embora a teoria do capital humano pressuponha sua importância para dinamizar o empreendedorismo, alguns vieses podem surgir, dependendo das características culturais dos indivíduos. Por exemplo, um indivíduo com uma forte formação educacional pode ser influenciado ao emprego formal e desencorajado a assumir riscos, ao passo que outro indivíduo com menos formação terá maior propensão a se envolver em atividades empresariais, em busca de ascendência social, exatamente para compensar a falta de investimentos em capital humano formal.

Diante das dificuldades de um entendimento mais preciso sobre o impacto do capital humano no empreendedorismo, o presente estudo concorda com o argumento de que sua importância pode ser diferente, dependendo do estágio de desenvolvimento do país. Trata-se de uma observação similar à apresentada na pesquisa de Barros e Pereira (2008), que relativizam a teoria do empreendedorismo pelo transbordamento do conhecimento (knowledge spillover theory of entrepreneurship) desenvolvida por Audretsch, Keibach e Lehmann (2006). Para esses autores, essa teoria foi formalizada sob a óptica dos países desenvolvidos, entretanto há carência de pesquisas no contexto de países com enorme contingente de mão de obra na economia informal, como é o caso do Brasil.

\section{DESENVOLVIMENTO ECONÔMICO E EMPREENDEDORISMO}

Na década de I990, alguns economistas identificaram a contribuição do empreendedorismo para o crescimento e desenvolvimento econômico, porém se limitaram ao contexto regional, setorial e/ou empresarial. Um dos trabalhos foi desenvolvido por Reynolds, Storey e Westhead (I994), no qual fazem uma comparação entre as taxas de criação de novas empresas em várias regiões de países europeus. Nesse estudo, os autores concluíram que a criação de empresas nos vários países é influenciada pelo engajamento do governo no apoio ao empreendedorismo, mediante incentivos à concepção de novos projetos de empresas, o que pode ser obtido por meio de mudanças nas leis e instituições; do oferecimento de facilidades para a gestação de novas empresas (por exemplo, fornecendo infraestruturas, disponibilizando cursos de formação); pelo apoio à sobrevivência e crescimento das start-ups, por meio do acesso às redes de informação, da disponibilização de consultoria, da criação de incubadoras e de outros recursos.

Carree e Thurik (I996) estudaram o efeito da iniciativa empresarial nas economias desenvolvidas e nos países em desenvolvimento. Eles concluíram que a 
presença de pequenas e médias empresas (PME) beneficia comparativamente de forma mais intensiva os países europeus mais desenvolvidos do que os países com renda per capita mais baixa, como Portugal e Espanha. Esses esforços em investigar empiricamente o impacto do "espírito empreendedor" na economia se limitaram ao âmbito da empresa, de regiões ou por setor, enquanto as análises comparativas entre países são bem escassas (VAN STEL; CARREE; THURIK, 2005). A literatura reconhece claramente os benefícios do empreendedorismo para o crescimento e desenvolvimento econômico. No entanto, essa visão está mais apoiada em bases teóricas do que em análises empíricas.

Uma das maiores dificuldades para fazer uma análise empírica da relação entre empreendedorismo e o desenvolvimento econômico de países e regiões é a obtenção de medidas adequadas. Existem algumas proposições práticas de medida do empreendedorismo, porém não são suficientemente boas medidas de proxy para o empreendedorismo no contexto schumpeteriano descrito anteriormente.

Uma importante contribuição na literatura são os trabalhos de Carree e Thurik (2003), onde são apresentadas diversas análises da contribuição do empreendedorismo para o crescimento e desenvolvimento econômico.

A Organização para a Cooperação e Desenvolvimento Econômico (OCDE, I998) reconhece que medir o empreendedorismo é uma tarefa muito difícil, pois não há consenso sobre o que seria o conjunto de indicadores práticos e confiáveis.

Barros e Pereira (2008) destacam que existem, pelo menos, quatro medidas de empreendedorismo: a primeira considera o número de trabalhadores por conta própria ou de proprietários de empresas; a segunda focaliza somente os novos negócios; a terceira incorpora à segunda medida os empreendedores no processo de criação do novo negócio: o empreendedorismo nascente na definição do GEM; a quarta medida de empreendedorismo é a participação das pequenas empresas na produção ou no emprego.

Sobre os estudos que usaram o número de trabalhadores por conta própria como proxy para o empreendedorismo, destacam-se os trabalhos de Audretsch e Thurik (200I), Blanchflower (2000), Blanchflower et al. (200I), Bruce e HoltzEakin (200I) e Carree, Klomp e Thurik (2000).

Earle (2000) critica o uso dessa medida e argumenta que podem fazer parte do grupo de trabalhadores por conta própria autônomos empreendedores que exploram novas oportunidades e inovam melhorando a produção, os processos de produção e as formas da distribuição, bem como indivíduos que, em meio às suas incapacidades, buscam soluções satisfatórias para que possam se tornar trabalhadores regulares. No primeiro caso, os autônomos se esforçam para aumentar e enriquecer seu negócio, e, para isso, correm riscos com novos 
empreendimentos, enquanto, no segundo caso, estão os indivíduos que buscam garantir sua sobrevivência. Conforme sugerido por Earle (2000), o número de trabalhadores por conta própria pode ser representado por um "empreendedorismo induzido" ou pelo "impulso do desemprego".

Apesar das mencionadas limitações da representação do empreendedorismo pela atividade dos trabalhadores por conta própria, a adoção dessa medida neste estudo justifica-se pela ausência de outras medidas em nível municipal e pela facilidade de acesso aos dados do Censo Demográfico de 2000 (IBGE, 2000).

\section{METODOLOGIA}

\subsection{QUESTÕES, DADOS E OBJETIVOS DA PESQUISA}

$\mathrm{Na}$ presente pesquisa, houve o interesse particular em investigar quatro questões básicas:

- Qual é a influência do capital humano para explicar as diferenças das taxas de desenvolvimento econômico entre os municípios?

- Qual é a contribuição do empreendedorismo para o desenvolvimento econômico dos municípios?

- É possível identificar uma relação entre o capital humano e o desenvolvimento econômico?

- Quais são as contribuições do capital humano, empreendedorismo e crescimento para o desenvolvimento econômico dos municípios?

Para responder a essas questões, foram definidos e obtidos os dados para as seguintes variáveis: empreendedorismo, capital humano, crescimento econômico e desenvolvimento econômico.

A variável dependente adotada pela presente pesquisa é o "desenvolvimento econômico", sendo representado pelo Índice de Desenvolvimento Municipal (IDM), estimado para o ano de 2006 pelo Instituto de Pesquisa e Estratégia Econômica do Ceará (Ipece). O IDM é calculado com base em um conjunto de trinta indicadores sociais, demográficos, econômicos e de infraestrutura de apoio referentes aos I84 municípios cearenses e permite a hierarquização dos municípios do Estado do Ceará, segundo seus níveis de desenvolvimento medidos, discriminados a seguir: grupo I (GI) - indicadores fisiográficos, fundiários e agrícolas; grupo $2(\mathrm{G} 2)$ - indicadores demográficos e econômicos; grupo $3\left(\mathrm{G}_{3}\right)$ - indicadores infraestrutura de apoio; e grupo $4\left(\mathrm{G}_{4}\right)$ - indicadores sociais. 
O "empreendedorismo" foi medido pela proporção dos trabalhadores por conta própria na população economicamente ativa com base nos dados municipais do Censo Demográfico do IBGE do ano 2000. O IBGE (2000, p. I2) define conta própria como sendo a "pessoa que trabalha explorando o seu empreendimento, sem empregados, individualmente ou com sócio, com o auxílio ou não de trabalhador remunerado membro da unidade domiciliar".

Para determinar o capital humano de cada um dos municípios cearenses, utilizou-se do procedimento de análise fatorial (AF) no grupo de variáveis denominado $\mathrm{G}_{4}$ (IPECE, 2008) para determinar uma proxy que representasse adequadamente a variável "capital humano".

A variável "crescimento econômico" foi definida pela taxa de crescimento do PIB per capita, sendo calculada a partir das estatísticas do Produto Interno Bruto (PIB) dos municípios cearenses publicadas pelo Anuário Estatístico do Ceará para o período de 2000 a 2003.

\subsection{HIPÓTESES DA PESQUISA}

A formulação das hipóteses da pesquisa visa alcançar os objetivos propostos e centra-se nas variáveis que ajudam a explicar o processo de desenvolvimento econômico dos municípios cearenses. Desse modo, estabeleceram-se as seguintes hipóteses:

- Hr: Os indicadores de capital humano exercem uma influência positiva sobre o crescimento econômico dos municípios cearenses.

- H2: Os indicadores de capital humano estão positivamente correlacionados com o desenvolvimento econômico dos municípios cearenses.

- H3: A relação entre empreendedorismo e crescimento econômico inexiste nos municípios cearenses.

- H4: A relação entre empreendedorismo e desenvolvimento econômico inexiste nos municípios cearenses.

\subsection{MÉTODOS DE ANÁLISE}

Para o presente estudo, três tipos de análise foram utilizados: I. análise fatorial; 2. análise de regressão múltipla; e 3. modelo de equações estruturais.

\subsubsection{ANÁLISE FATORIAL}

A primeira parte do estudo consistiu na estimativa da variável "capital humano" para cada um dos municípios do Estado do Ceará. Apesar de autores como 
Romer (200I) associarem capital humano a escolaridade, nutrição e saúde da população, uma vasta literatura mede o capital humano tendo como proxy apenas a escolaridade (LEE, 200I; BACHA, 2004; ROCHA, 2008).

Inicialmente, utilizou-se o grupo de variáveis denominado $\mathrm{G}_{4}$ (IPECE, 2008) para determinar uma proxy que representasse adequadamente a variável "capital humano". Tal grupo foi composto das variáveis seguintes: taxa de escolarização no ensino médio em 2006 (ESC), taxa de aprovação no ensino fundamental em 2005 (APR), número de equipamentos de informática por escola em 2006 (INF), biblioteca, salas de leitura e laboratórios de informática por escola em 2006 (BIB), e proporção de docentes no ensino fundamental com formação superior em 2006 (DOC).

Com o propósito de criar uma representação para o capital humano, adotouse o procedimento de análise fatorial (AF) (utilizando o método dos componentes principais), técnica que, segundo informam Hair Jr. et al. (2005, p. 9I), consiste em uma "classe de métodos multivariados cujo propósito principal é definir a estrutura subjacente em uma matriz de dados”. A partir dessa técnica, foi possível verificar quais as variáveis do grupo $4\left(\mathrm{G}_{4}\right)$ dos indicadores sociais estavam consistentemente associados ao construto "capital humano". Após os procedimentos, para cada um dos construtos foi extraído o alfa de Cronbach, para melhor avaliar a confiabilidade da mensuração do construto definido (MALHOTRA, 200I). Após esses procedimentos, e confirmada a estrutura dos itens na composição do construto, foi extraída uma medida geral na forma de escore fatorial. Adicionalmente, considerando que as hipóteses supõem relacionamentos entre construtos, decidiu-se avaliar esses relacionamentos por meio da ferramenta análise de regressão múltipla, que viabiliza a avaliação da consistência da relação de influência entre duas ou mais variáveis independentes e uma variável dependente (MALHOTRA, 200I).

\subsubsection{ANÁLISE DE REGRESSÃO}

Para testar as quatro hipóteses, foram realizados os seguintes procedimentos:

I. Estabeleceu-se relação entre o IDM como variável dependente e as variáveis capital humano $(\mathrm{CH})$, empreendedorismo (TE) e crescimento econômico (CE). Utilizou-se a técnica stepwise como forma de verificar as variáveis excluídas ou incluídas no modelo de regressão, o que contribuiu para o entendimento de como o empreendedorismo se relaciona com o desenvolvimento econômico nesse Estado. Conforme pode ser constatado, o processo de ajuste desse método para uma variável dependente e $k$ variáveis independentes baseia-se em iterações sucessivas, com a inclusão e exclusão de 
variáveis controladas por um nível de significância adotado, o qual em geral é medido pela estatística F-Snedecor (MENDENHALL; SINCICH, I993).

2. Foi também realizada regressão stepwise entre o crescimento econômico (CE) como variável dependente e as preditoras capital humano $(\mathrm{CH})$, empreendedorismo (TE) e desenvolvimento econômico (IDM), com o propósito de conhecer o papel do empreendedorismo especificamente em relação ao crescimento econômico, o que serviu para reforçar os resultados obtidos no item anterior.

3. Finalmente, foram relacionadas todas as variáveis em conjunto, a partir dos respectivos construtos, na forma de modelo de equações estruturais, o que possibilitou conhecer o efeito das variáveis empreendedorismo (TE) e capital humano $(\mathrm{CH})$ dentro de uma perspectiva integrada. De fato, esse último tratamento quantitativo permitiu analisar tanto o crescimento como o desenvolvimento econômico na forma de variáveis dependentes simultâneas, o que contribuiu sobremaneira para conhecer melhor o impacto do empreendedorismo e o capital humano nos municípios do Ceará.

Para o presente trabalho, além da obtenção dos modelos preditores do desenvolvimento e crescimento dos municípios, também é de relevância a investigação sobre quais variáveis são inseridas ou excluídas do modelo. Por essa razão é que foi preferida a técnica stepwise, em detrimento das correspondentes backward e forward. Adotaram-se as probabilidades de entrada e saída no modelo, respectivamente de 0,05 e o,Io, que correspondem ao padrão do SPSS v.I7, que foi o software adotado para o processamento das informações. A técnica stepwise consiste em um processo sequencial de inclusão de variáveis preditoras, uma a uma, selecionando a cada passo a que apresenta maior contribuição para explicar a variável dependente e, eventualmente, descartando variáveis selecionadas em passos anteriores se atingirem o nível crítico de significância. O procedimento chega ao fim quando não há mais nenhuma variável não incluída que seja capaz de contribuir significativamente e nenhuma variável incluída que deva ser descartada.

\subsubsection{MODELO DE EQUAÇÕES ESTRUTURAIS}

Tendo por objetivo a integração dos construtos do estudo e análise de suas relações, utilizou-se a modelagem de equações estruturais (SEM), uma vez que se trata de uma técnica que combina aspectos na regressão múltipla, com a análise fatorial (HAIR JR. et al., 2005).

A elaboração do modelo seguiu uma abordagem exploratória, que é reconhecida por Jöreskog e Sörbom (I999) como abordagem de geração de modelos. A modelagem de equações estruturais pode ser utilizada para propósitos confir- 
matórios e para projetos exploratórios (ANDERSON; GERBING, I988; KLINE I998). Segundo Anderson e Gerbing (I988), o limite entre as abordagens exploratórias e confirmatórias não é claro, uma vez que a maioria dos modelos utilizados em análise confirmatória não é ajustada imediatamente. Nesses casos, esses modelos não são descartados, sendo sugeridas modificações que caracterizam a investigação como de caráter exploratório.

Outra técnica utilizada na verificação desse modelo foi a de bootstrapping, que consiste na repetição de amostragens originais, para efeito de comparação dos resultados com o que foi obtido na amostra utilizada. Dessa forma, o modelo estrutural integrou os construtos capital humano $(\mathrm{CH})$ e empreendedorismo (TE) com crescimento econômico (CE) e desenvolvimento econômico (IDM).

\section{ANÁLISE dos RESULTAdos}

\subsection{CONSTRUÇÃO DO ÍNDICE DE CAPITAL HUMANO}

Com o propósito de criar uma representação para o capital humano, adotouse o procedimento de análise fatorial (AF) (utilizando o método dos componentes principais), que gerou os seguintes resultados: medida de adequação amostral KMO: 0,698 ; teste de esfericidade de Bartlett e $\chi_{2}^{2}$ aproximado de IoI, com ıо graus de liberdade e significância $\circ$.

Utilizou-se uma amostra de 184 registros, correspondentes aos municípios do Estado do Ceará, o que resultou em mais de 20 registros por variável, suficientes para a realização do procedimento da AF. Ademais, o número de correlações em torno de 0,30 foi expressivo dentre os pares das variáveis escolhidas. Isso pode ser atestado pelo teste de Bartlett já mencionado, que rejeitou a hipótese nula da ausência de correlações entre as variáveis, apontando para a existência de correlações significativas em pelo menos algumas das variáveis (HAIR JR., 2005, p. 98). A outra medida de adequação amostral (KMO) obtida também indica que o fator representativo das cinco variáveis, baseado na $\mathrm{AF}$, decerto será válido para representar o grupo das variáveis originais.

Como o principal propósito dessa etapa foi estabelecer um número mínimo de fatores para explicar a maior porção da variância do conjunto, adotou-se o método dos componentes principais, que resultou em um único componente (fator) com autovalor de 2,02 - portanto superior a I - e que explicou 40,4\% da variação total do grupo. As cargas fatoriais (factor loadings), representativas da correlação entre cada variável analisada e o principal componente, encontram-se na Tabela I. 


\section{TABELA I}

CORRELAÇÃO ENTRE VARIÁVEIS

\begin{tabular}{lc}
\hline VARIÁVEIS COMPONENTES DO G4 DO IDM (2006) & COMPONENTE PRINCIPAL \\
\hline Taxa de escolarização no ensino médio em 2006 (ESC) & 0,619 \\
\hline Taxa de aprovação no ensino fundamental em 2005 (APR) & 0,528 \\
\hline Número de equipamentos de informática por escola em 2006 (INF) & 0,712 \\
\hline $\begin{array}{l}\text { Biblioteca, salas de leitura e laboratórios de informática por escola } \\
\text { em } 2006 \text { (BIB) }\end{array}$ & 0,732 \\
\hline $\begin{array}{l}\text { Proporção de docentes no ensino fundamental com formação } \\
\text { superior em } 2006 \text { (DOC) }\end{array}$ & 0,561 \\
\hline
\end{tabular}

Fonte: Elaborada pelos autores.

As magnitudes das cargas fatoriais permitem definir as variáveis mais representativas do fator associado. No caso presente, os maiores valores foram de 0,732 para a variável "biblioteca, salas de leitura e laboratórios de informática por escola em 2006 (BIB)" e de 0,7I2 para "número de equipamentos de informática por escola em 2006 (INF)". Como se pode observar, esses valores são expressivamente maiores que os demais, o que os habilita para denominar o fator aqui extraído.

A análise das cargas fatoriais mostra, portanto, que as principais variáveis representadas por esse único fator são aquelas associadas com a infraestrutura escolar, quais sejam, INF e BIB, representativas de locais e equipamentos usados nas escolas dos municípios. Na sequência, obtiveram-se os escores fatoriais, os quais consistem nos valores em escala normalizada associados ao fator extraído, doravante denominado de capital humano $(\mathrm{CH})$ e que será utilizado posteriormente para analisar as relações com as demais variáveis julgadas relevantes para o estudo.

\subsection{ANÁLISE CORRELACIONAL}

Como citado na metodologia, buscou-se conhecer o efeito do capital humano $(\mathrm{CH})$ e empreendedorismo (TE) sobre o desenvolvimento (IDM) e crescimento econômico (CE). Assim, relacionaram-se as variáveis CE e IDM com CH e TE na forma de duas regressões stepwise, em que a contribuição de cada variável é avaliada pelo grau da sua respectiva significância (medida pela estatística F), resultando em um modelo formado pelas variáveis mais relevantes. 
Com o objetivo de captar a influência do capital humano, empreendedorismo e crescimento econômico para o desenvolvimento econômico dos municípios cearenses, optou-se, preliminarmente, por efetuar a regressão pelo método stepwise, onde IDM $=\mathrm{f}(\mathrm{CH}, \mathrm{TE}, \mathrm{CE})$.

A Tabela 2 apresenta quais variáveis foram excluídas por não apresentarem relevância com o desenvolvimento econômico.

\section{TABELA 2}

VARIÁVEIS EXCLUÍDAS

IDM DEPENDENTE E (CH, TE, CE) INDEPENDENTES

\begin{tabular}{lccc}
\hline MODELO & COEFICIENTES $\beta$ & $\mathrm{t}$ & SIG. \\
\hline Taxa de empreendedorismo (TE) & $-0,040$ & $-0,71$ & 0,479 \\
\hline Crescimento econômico (CE) & 0,064 & 1,113 & 0,267 \\
\hline
\end{tabular}

Fonte: Elaborada pelos autores.

A Tabela 2 mostra que as variáveis TE e CE não se mostraram relevantes para explicar o desenvolvimento municipal (IDM), em virtude de um baixo poder explicativo dos coeficientes da regressão, como indicado pelos $\beta$, os quais não detêm significância estatística.

Ao se considerar, porém, o crescimento econômico (CE) como variável dependente, o modelo fica representado por $\mathrm{CE}=\mathrm{f}(\mathrm{CH}, \mathrm{TE}$, IDM) e, após aplicação da técnica stepwise, a exemplo do caso anterior, demonstra quais variáveis não tiveram relevância para explicar o crescimento econômico (Tabela 3).

\section{TABELA 3}

\section{VARIÁVEIS EXCLUÍDAS}

CE DEPENDENTE E (CH, TE, IDM) INDEPENDENTES

\begin{tabular}{lccc}
\hline MODELO & COEFICIENTES $\beta$ & $t$ & SIG. \\
\hline Taxa de empreendedorismo (TE) & $-0,020$ & $-0,271$ & 0,787 \\
\hline Desenvolvimento econômico (IDM) & 0,108 & 1,113 & 0,267 \\
\hline
\end{tabular}

Fonte: Elaborada pelos autores.

Os resultados provenientes das tabelas 2 e 3 de certa forma reforçam a hipótese de que a capacidade empreendedora no Estado tem consistido apenas em mais uma alternativa de trabalho e não em uma contribuição para o desenvol- 
vimento e crescimento econômicos. Além disso, os dados confirmam os resultados obtidos em pesquisas anteriores (VAN STEL; CARREE; THURIK, 2005; BARROS; PEREIRA, 2008), que revelam uma associação negativa entre a atividade empreendedora e o crescimento econômico.

Após a exclusão das variáveis não significativas, as equações baseadas no modelo de regressão ficaram:

$$
\begin{gathered}
I D M=28,191+7,465 C H \\
C E=7,99+0,832 C H
\end{gathered}
$$

As referidas equações permitem observar que, ao contrário do empreendedorismo, o capital humano é relevante tanto para explicar o crescimento como o desenvolvimento econômico, tal como obtiveram os trabalhos de Oliveira Silva (2006), Oliveira (2006), Barreto e Almeida (2009) e Lima et al. (2009).

A Tabela 4 sumariza os coeficientes e índices dos modelos de regressão adotados.

\section{TABELA 4}

RESUMO DA REGRESSÃO STEPWISE PARA AMBOS OS MODELOS VARIÁVEL INDEPENDENTE: CH

\begin{tabular}{lcc}
\hline COEFICIENTES & MODELO 1 & MODELO 2 \\
& VAR. DEPENDENTE IDM & VAR. DEPENDENTE CE \\
\hline Correlação (R) & 0,67 & 0,23 \\
\hline Determinação (R2 ajustado) & 0,44 & 0,05 \\
\hline Anova & & \\
• F & 142,77 & 9,66 \\
• Significância & 0,00 & 0,002 \\
\hline Coeficiente de regressão (variável CH) & & \\
• Não padronizado (b) & 7,5 & 0,83 \\
• t & 11,95 & 3,11 \\
• Significância & 0,000 & 0,002 \\
• $\beta$ & 0,67 & 0,23 \\
\hline
\end{tabular}

Fonte: Elaborada pelos autores.

Em ambos os modelos, a variável capital humano $(\mathrm{CH})$ apresentou relação positiva com as variáveis dependentes, reforçando a importância do nível de co- 
nhecimento para um maior crescimento e desenvolvimento econômico. O coeficiente não padronizado indica que um aumento em uma unidade no escore fatorial representativo do $\mathrm{CH}$ eleva o IDM em quase 7,5 unidades, assim como aumenta o crescimento em 0,83 unidade.

Percebe-se que, enquanto o $\mathrm{CH}$ mostra um razoável grau de relação com o IDM $(0,67)$, a correspondente correlação com CE já é consideravelmente menor $(0,23)$, embora sejam ambos significantes em níveis inferiores a o,०I, como atestam os valores referentes à análise de variância (Anova) correspondente.

No que se refere à capacidade de previsão, observa-se que o R2 ajustado de 0,44 indica que o modelo baseado no capital humano consegue explicar em $44 \%$ o desenvolvimento econômico dos municípios cearenses. O crescimento econômico, por sua vez, é praticamente impossível de ser previsto a partir daquela variável, uma vez que o seu correspondente coeficiente de determinação só chegou a 0,05 . Entretanto, as estatísticas F-Snedecor (respectivamente de 142,77 e 9,66) referentes à Anova indicaram que os resultados obtidos por esse modelo possuem significância estatística, isto é, não se apoiam apenas em flutuações ocasionais, podendo ser estendidos para uma população como um todo.

\subsection{AVALIAÇÃO DO MODELO ESTRUTURAL}

Com o objetivo de avaliar o modelo identificado na revisão de literatura, que relacionou o capital humano $(\mathrm{CH})$ e taxa de empreendedorismo (TE) com o desenvolvimento municipal (IDM) e o crescimento econômico (CE), utilizou-se a técnica de modelagem de equação estrutural.

Optou-se pela utilização do software SmartPLS 2.0 para modelagem estrutural. Para cada construto, considerou-se a análise fatorial realizada, de forma que os indicadores foram agrupados em cada fator, em razão da significância das cargas fatoriais. Inicialmente, analisou-se cada construto isoladamente, e os critérios utilizados na análise foram: os coeficientes encontrados, o alfa de Cronbach e a validade convergente. Foram considerados satisfatórios: as cargas acima de 0,7 , a confiabilidade acima de 0,7 e a variância explicada (AVE) acima de 0,5 .

Inicialmente, avaliaram-se as variáveis para verificação da validade discriminante. Avalia-se a validade discriminante comparando a raiz da AVE com as correlações. Espera-se que a raiz da AVE de cada construto seja maior que as correlações entre os construtos. Para tanto, calculou-se a raiz da AVE, sendo apresentada na coluna diagonal da matriz de correlações, conforme Tabela 5. Percebe-se que o modelo apresenta validade discriminante para todas as variáveis de primeira ordem, o que representa um resultado satisfatório. 


\section{TABELA 5}

MATRIZ DE VALIDADE DISCRIMINANTE

\begin{tabular}{lcccc}
\hline & TE & CH & IDM & CE \\
\hline TE & 1 & 0 & 0 & 0 \\
\hline CH & 0,021 & 0,838212 & 0 & 0 \\
\hline IDM & $-0,0301$ & 0,5789 & 0,770065 & 0 \\
\hline CE & $-0,0468$ & 0,2626 & 0,133 & 1 \\
\hline
\end{tabular}

Fonte: Elaborada pelos autores.

Dessa forma, os resultados do modelo estrutural são apresentados na Figura I.

\section{FIGURA I}

\section{MODELO ESTRUTURAL PARA CRESCIMENTO E \\ DESENVOLVIMENTO MUNICIPAL COM BASE NO CAPITAL HUMANO E EMPREENDEDORISMO}

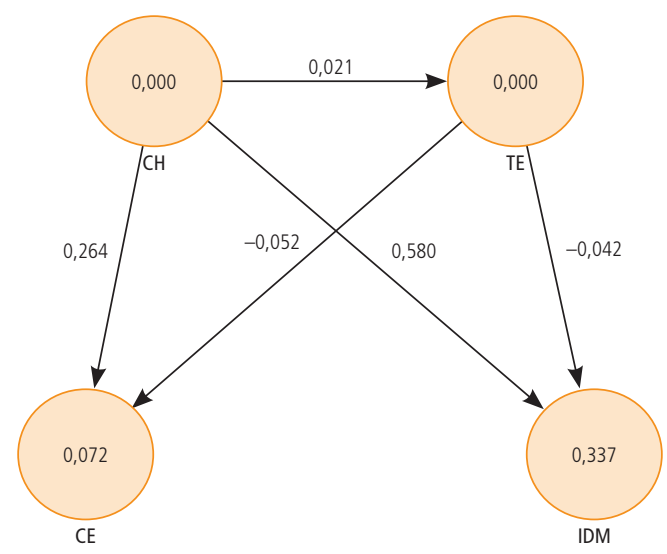

Fonte: Elaborada pelos autores.

Quando se analisam os coeficientes paths, são identificados resultados com pouca significância em alguns construtos: no construto empreendedorismo, percebe-se que a relação com o crescimento mostrou-se pouco significativa $(-0,052)$, o que também aconteceu com o desenvolvimento municipal $(-0,004)$. Esse resultado colabora com a literatura, uma vez que são apontadas limitações 
na relação empreendedorismo e crescimento econômico (OCDE, I998; EARLE, 2000), principalmente em países não desenvolvidos (CARREE; THURIK, I996). O construto capital humano, por sua vez, apresentou um resultado mais expressivo, na relação com o crescimento $(0,264)$ e com o desenvolvimento municipal $(0,580)$. Assim, o resultado mais significativo obtido foi o do desenvolvimento municipal, sendo explicado pelos construtos capital humano e capacidade empreendedora, com um R2 de 0,337 .

\section{TABELA 6}

ÍNDICES DE DESEMPENHO DO MODELO

\begin{tabular}{lcccc}
\hline & AVE & CONFIABILIDADE & R2 & COMUNALIDADE \\
\hline TE & 1 & 1 & 0,0004 & 1 \\
\hline CH & 0,7026 & 0,8237 & 0 & 0,7026 \\
IDM & 0,593 & 0,8017 & 0,3369 & 0,593 \\
\hline CE & 1 & 1 & 0,0717 & 1 \\
\hline
\end{tabular}

Fonte: Elaborada pelos autores.

Com o propósito de analisar se o modelo apresentado está ajustado à amostra utilizada, fez-se uso do método bootstrapping. Trata-se de um método com amplo poder computacional em que repetidas subamostras são retiradas da base de dados original, e os parâmetros de interesse, calculados. Para tanto, definiu-se o cálculo para 200 subamostras, cujos resultados são apresentados na Tabela 7 .

\section{TABELA 7}

\section{INDICES DO MÉTODO BOOTSTRAPPING}

\begin{tabular}{lccccc}
\hline & AMOSTRA ORIGINAL & MÉDIA AMOSTRAS & (STDEV) & STAND ERRO & TESTE T \\
\hline $\mathrm{TE} \rightarrow$ IDM & $-0,0423$ & $-0,0447$ & 0,0531 & 0,0531 & 0,7971 \\
\hline $\mathrm{TE} \rightarrow \mathrm{CE}$ & $-0,0523$ & $-0,0559$ & 0,0534 & 0,0534 & 0,9793 \\
\hline $\mathrm{CH} \rightarrow \mathrm{TE}$ & 0,021 & 0,0233 & 0,0582 & 0,0582 & 0,3615 \\
\hline $\mathrm{CH} \rightarrow \mathrm{IDM}$ & 0,5789 & 0,5594 & 0,0903 & 0,0903 & 6,4135 \\
\hline $\mathrm{CH} \rightarrow \mathrm{CE}$ & 0,2626 & 0,2817 & 0,0591 & 0,0591 & 4,4412 \\
\hline
\end{tabular}

Fonte: Elaborada pelos autores. 
Os resultados obtidos pelo método bootstrapping mostram que a média obtida nas 200 subamostras, para cada relação entre os construtos, são próximas da média da amostra original.

Com base nos resultados obtidos pelo modelo estrutural, faz-se necessário apontar o teste de hipóteses: um parâmetro observável é a estatística T, com predominância de valores superiores a I,96. Pelo teste T, as relações que se confirmaram estatisticamente no modelo foram: capital humano $(\mathrm{CH}) \mathrm{com}$ o desenvolvimento municipal (IDM) e do capital humano $(\mathrm{CH})$ com o crescimento $(\mathrm{CE})$.

Dessa forma, a hipótese Hi - Os indicadores de capital humano exercem uma influência positiva sobre o crescimento econômico dos municípios cearenses - foi confirmada pelo índice do teste $\mathrm{T}(4,44 \mathrm{I} 2)$, tendo um coeficiente path positivo de $(0,264)$.

$\mathrm{O}$ coeficiente path relacionado à hipótese $\mathrm{H}_{2}$ - Os indicadores de capital humano estão positivamente correlacionados com o desenvolvimento econômico dos municípios cearenses - foi positivo em 0,580 , o que apoiado pelo teste $\mathrm{T}(6,4 \mathrm{I} 35)$, o que confirma a hipótese.

A hipótese $\mathrm{H}_{3}-\mathrm{A}$ relação entre empreendedorismo e crescimento econômico inexiste nos municípios cearenses - foi confirmada, uma vez que o coeficiente path foi negativo e o teste $\mathrm{T}$ demonstrou a inexistência de relação $(0,9793)$.

Da mesma forma, o índice obtido no teste T (0,797I) confirma a hipótese $\mathrm{H}_{4}$ - A relação entre empreendedorismo e desenvolvimento econômico inexiste nos municípios cearenses.

Os dados obtidos no modelo estão coerentes com os resultados da análise de regressão múltipla realizada, confirmando a existência de relação do capital humano $(\mathrm{CH})$ com o crescimento econômico (CE) e o desenvolvimento municipal (IDM), bem como pela inexistência de relação estatisticamente significante entre a taxa de empreendedorismo com o crescimento econômico (CE) e o desenvolvimento municipal (IDM).

\section{CONCLUSÕES}

O presente estudo buscou ampliar o conhecimento sobre os determinantes do desenvolvimento econômico dos municípios cearenses. Nesse aspecto, verificou-se que o capital humano contribui positivamente para o crescimento e desenvolvimento econômico dos municípios, corroborando os resultados de outros estudos como o de Oliveira (2006), Oliveira Silva (2006), Irffi et al. (2009) e Barreto e Almeida (2009). O resultado da pesquisa revela que, para promover 
o desenvolvimento econômico municipal, devem-se criar mecanismos para favorecer a criação de capital humano - que viria com melhores investimentos em educação -, no intuito de gerar efeitos qualitativos na economia. Esse efeito qualitativo acaba gerando spillovers sobre a melhoria das instituições do Estado, bem como na geração de melhores negócios, consequentemente gerando um círculo virtuoso na economia e contribuindo para uma melhor distribuição de renda.

No que se refere aos resultados que demonstram que o empreendedorismo se apresenta insignificante estatisticamente para compreender as diferenças de crescimento econômico dos municípios, os autores ressalvam algumas limitações metodológicas em estudos dessa natureza. É evidente que a variável empreendedorismo, medida pela proporção de trabalhadores por conta própria, pode não representar corretamente o dinamismo da economia local, visto que esses trabalhadores são constituídos em sua grande maioria por indivíduos de baixa produtividade e renda, que buscam uma ocupação alternativa ao desemprego, conforme Earle (2000). Porém, apesar das limitações da medida do empreendedorismo pela atividade dos trabalhadores por conta própria, os resultados do estudo confirmam conclusões obtidas em outros trabalhos: o empreendedorismo pode ser diferente, dependendo do estágio de desenvolvimento do país (AGHION; HOWITT, 2005), e o impacto do empreendedorismo sobre as taxas de crescimento econômico é negativo (BARROS; PEREIRA, 2008).

Como pesquisa futura, sugere-se a construção de um modelo mais amplo que contemple outras variáveis explicativas, possibilitando ampliar o conhecimento do papel do empreendedorismo e do capital humano no desenvolvimento econômico. Outra possibilidade seria a extensão da pesquisa fazendo comparações com outros Estados da Federação e/ou entre países, bem como replicar este estudo longitudinalmente em outros anos.

\section{HUMAN CAPITAL, ENTREPRENEURSHIP AND DEVELOPMENT: EMPIRICAL EVIDENCE IN THE MUNICIPALITIES OF CEARÁ}

\section{ABSTRACT}

The contribution of human capital to economic development of nations has been the subject of several studies since the I96os. However, updating the theme started in the second half of the ' 90 s, with the examination of the role of entrepreneurship is the main contribution of this study. Thus, this article falls within the context of this update on a subject not explored in the academy at a 
reasonable extent. Therefore, this article investigates the influence of human capital and entrepreneurship on economic development. More specifically, the article analyzes the influence of the two variables on improving the quality of life of cities in the state of Ceará, Brazil. We gathered a database of social indicators, demographic, and economic infrastructure, of all I84 municipalities of Ceará. This secondary data was provided by the Instituto de Pesquisa e Estratégia Econômica do Ceará (Ipece) and the Instituto Brasileiro de Geografia e Estatística (IBGE). We setup three analytical techniques to confirm the hypotheses formulated based on published literature: factor analysis, regression analysis and structural equation model. The results supported the hypothesis that entrepreneurship in the state of Ceará has consisted only in an alternative possibility and not in a contribution to development and economic growth. Despite the limitations of the measure of entrepreneurial activity by self-employed workers, the results confirm findings from other studies: entrepreneurship may be different, depending on the stage of development of the country (AGHION; HOWITT, 2005) and the impact of entrepreneurship on economic growth rates is negative (BARROS; PEREIRA, 2008). As future research, it is suggested the construction of a model that includes other explanatory variables that implies in increasing the knowledge of the role of entrepreneurship and human capital development. Another possibility would be to extend the research by making comparisons with other states in the country and/or between countries, as well as replicating this study to other years.

\section{KEYWORDS}

Human capital; Entrepreneurship; Economic growth; Economic development; Ceará.

\section{EL CAPITAL HUMANO, INICIATIVA EMPRESARIAL Y DESARROLLO: EVIDENCIA EMPÍRICA EN LOS MUNICIPIOS DE CEARÁ}

\section{RESUMEN}

La contribución del capital humano para el proceso de desarrollo económico de las naciones ha sido objeto de varios estudios desde la década de ig6o. Sin embargo, la actualización del tema se inició en la segunda mitad de los años 90 con la necesidad de examinar el papel del espíritu empresarial en este proce- 
so. Estas investigaciones se inscriben en el contexto de esta actualización, sobre un tema tan poco explorado en la Academia. Por lo tanto, este artículo estudia la influencia del capital humano y el espíritu empresarial en el proceso de desarrollo económico. Más concretamente, el artículo analiza su influencia en la mejora de la calidad de vida de las ciudades en el Estado de Ceará. Para el estudio, se reunieron una base de datos de indicadores sociales, demográficos, y la infraestructura económica de los I84 municipios de Ceará, a partir de datos secundarios proporcionados por el Instituto de Pesquisa e Estratégia Econômica do Ceará (Ipece) y el Instituto Brasileiro de Geografia e Estatística (IBGE) y hubo tres técnicas analíticas para confirmar las hipótesis formuladas sobre la base de la literatura publicada: análisis factorial, análisis de regresión y un modelo de ecuaciones estructurales. Los resultados del análisis apoyan la hipótesis de que el espíritu empresarial en este estado ha consistido sólo en un trabajo alternativo y no como una contribución al desarrollo y el crecimiento económico. A pesar de las limitaciones de la medida de la actividad empresarial por los trabajadores autónomos, los resultados del estudio confirman los hallazgos de otros estudios: el espíritu empresarial puede ser diferente dependiendo de la etapa de desarrollo del país (AGHION; HOWITT, 2005) lo que afirma que el impacto de la iniciativa empresarial en las tasas de crecimiento econômico es negativo y el impacto de la iniciativa empresarial en las tasas de crecimiento económico es negativo (BARROS; PEREIRA, 2008). Como investigación futura, se sugiere la construcción de un modelo que incluye otras variables explicativas, por lo que es posible ampliar el conocimiento del papel del espíritu empresarial y el desarrollo del capital humano. Otra posibilidad sería la de extender la investigación al hacer comparaciones con otros estados de la federación y/o entre los países, así como a lo largo de replicar este estudio en otros años.

\section{PALABRAS CLAVE}

Capital humano; Espíritu empresarial; Crecimiento económico; Desarrollo económico; Ceará.

\section{REFERÊNCIAS}

ACS, Z. J.; AUDRETSCH, D. B. Innovation and small firms. Cambridge: MIT Press, I990. AGHION, P.; HOWITT, P. Growth with quality-improving innovations: an integrated framework. Handbook of Economic Growth. Harvard University, 2005. Disponível em: <http://www.economics.harvard.edu/files/faculty/I_Growth_with_Quality.pdf>. Acesso em: 3 fev. 20 Iо. 
ANDERSON, J. C.; GERBING, D. W. Structural equation modeling in practice: a review and recommended two-steps approach. Psychological Bulletin, v. I03, n. 3, p. 4II-423, May I988.

AUDRETSCH, D. B.; KEIBACH, M. C.; LEHMANN, E. E. Entrepreneurship and economic growth. New York: Oxford University Press, 2006.

AUDRETSCH, D. B.; THURIK, A. R. What is new about the new economy: sources of growth in the managed and entrepreneurial economies. Industrial and Corporate Change, v. I0, n. I9, p. 2673I5, Nov. 200I.

BACHA, C. G. C. Macroeconomia aplicada à análise da economia brasileira. São Paulo: Edusp, 2004. BARRETO, R. C. S.; ALMEIDA, E. S. A contribuição do capital humano para crescimento econômico e convergência espacial do PIB per capita no Ceará. In: Economia do Ceará em Debate 2008. 4., 2008, Fortaleza. Anais... Fortaleza: Ipece, 2009.

BARRO, R. J. Economic growth in a cross section of countries. The Quarterly Journal of Economics, Cambridge, v. I06, n. 2, p. 407-43, May I99I.

BARRO, R. J.; LEE, J. W. International data on educational attainment: updates and implications. Oxford Economic Papers, v. 53, n. 3, p. 54I-56, Sept. 200 I.

BARROS, A. A.; PEREIRA, C. M. M. A. Empreendedorismo e crescimento econômico: uma análise empírica. Revista de Administração Contemporânea, v. I2, n. 4, p. 975-993, dez. 2008.

BARTLETT, C. A.; GHOSHAL, S. Strategic advantage change your mindset then shift direction. Executive Excellence, v. I9, Issue 7, p. 7-8, July 2002.

BECKER, G. S. Human capital: a theoretical and empirical analysis, with special reference to education. Chicago: The University of Chicago Press, I964. 4I2 p.

BENHABIB, J.; SPIEGEL, M. M. The role of human capital in economic development evidence from aggregate cross-country data. Journal of Monetary Economics, v. 34, n. 2, p. I43-I73, I994.

BLANCHFLOWER, D. G. Self-employment in OECD countries. Labour Economics, v. 7, Issue 5, p. 47I-505, Sept. 2000.

BLANCHFLOWER, D. G. et al. Latent entrepreneurship across nations. European Economic Review, v. 45, Issues 4-6, p. 680-69I, May 200 I.

BLEANEY, M.; NISHIYAMA, A. Explaining growth: a contest between models. Journal of Economic Growth, v. 7, p. 43-56, Mar. 2002.

BRUCE, D.; HOLTZ-EAKIN, D. Who are the entrepreneurs? Evidence from taxpayer data. Journal of Entrepreneurial Finance and Business Ventures, v. I, n. I, p. I-Io, May $200 \mathrm{I}$.

CARREE, M. A.; KLOMP, L.; THURIK, A. R. Productivity convergence in OECD manufacturing industries. Economic Letters, v. 66, p. 337-345, 2000.

CARREE, M. A.; THURIK, A. R. Industrial structure and economic growth. In: AUDRETSCH, D. B.; THURIK, A. R. (Ed.). Innovation, industry evolution and employment. Cambridge: Cambridge University Press, I996. p. 86-IIO.

The impact of entrepreneurship on economic growth. 2003. Disponível em: <http://www. ondernemerschap.nl/pdf-ez/N200320.pdf >. Acesso em: 2 fev. 2010.

DAVIDSSON, P. E.; HONIG, B. The role of social and human capital among nascent entrepreneurs. Journal of Business Venturing, v. I8, n. 3, p. 30I-330, May 2003.

DIAS, J.; DIAS, M. H. A. Crescimento econômico, emprego e educação em uma economia globalizada. Maringá: Eduem, I999. p. 89. 
DOWRICK, S. Ideas and education: level or growth effects? NBER Working Paper Series, Working Paper 9709, 2003. Disponível em: <http://www.nber.org/papers/w9709.pdf>. Acesso em: I5 abr. $20 I O$.

EARLE, P. The entrepreneur as a constructor of connections. 2000. Disponível em: <http://espace. uq.edu.au/eserv/UQ:9659/Earl_The_Entrepr.pdf>. Acesso em: i3 mar. 20 Io.

FRANK, O. O investimento em capital humano. 2008. Disponível em: <http://pensandoemeconomia. blogspot.com/2008/03/o-investimento-em-capital-humano.html> Acesso em: I6 abr. 2010.

HAIR JR., J. F. et al. Análise multivariada de dados. 5. ed. Porto Alegre: Bookman, 2005.

IBGE. Censo demográfico de 2000. Disponível em: <http://www.ibge.gov.br>. Acesso em: I2 jan. $20 I 0$.

IPECE - Instituto de Pesquisa e Estratégia Econômica do Ceará. 2008. Disponível em: <http:// www.ipece.gov.br>. Acesso em: 3 fev. 2010.

IRFFI, G. et al. Determinantes do crescimento econômico dos municípios cearenses, uma análise dos dados em painel. In: Economia do Ceará em debate 2008. Fortaleza: Ipece, 2009.

JÖRESKOG, K. G.; SÖRBOM, D. LISREL. 8: structural equation modeling with the SIMPLIS command language. Lincolnwood: Scientific Software International. I999.

KIRZNER, I. Competition and entrepreneurship. Chicago: University of Chicago Press, I973.

KLINE, R. B. Principles and practice of structural equation modeling. New York: Guilford Press, I998. KROTH, D. C.; DIAS, J. A contribuição do crédito bancário e do capital humano no crescimento econômico dos municípios brasileiros: uma avaliação em painéis de dados dinâmicos. In: ENCONTRO NACIONAL DE ECONOMIA, 34., 2006, Salvador. Anais... Bahia: Anpec, 2006. Disponível em: <http://www.anpec.org.br/encontro2006/artigos/Ao6AoI5.pdf>. Acesso em: 2 fev. 2010.

LEE, J. W. Education for technology readiness: prospects for developing countries. Journal of Human Development, v. 2, n. I, p.II5-I5I, 200I. Disponível em: <http://unpani.un.org/intradoc/ groups/public/documents/APCITY/UNPANo02133.pdf>. Acesso em: I2 out. 2009.

LIMA, P. V. P. S. et al. Capital humano no estado do Ceará: análise discriminante entre municípios. In: Economia do Ceará em Debate 2008. Fortaleza: Ipece, 2009.

LUCAS JR., R. E. On the mechanics of economic development. Journal of Monetary Economics, v. 22, p. 3-42, I988.

LUNDSTRÖM, L.; STEVENSON, A. Patterns and trends in entrepreneurship/sme policy and practice in ten economies. Swedish Foundation for Small Business Research, 200I. Disponível em: <http:// www.donnerenviedentreprendre.com/documentation/IMG/pdf/Volume_3_Chapitre_I.pdf>. Acesso em: I5 mar. 2010.

MALHOTRA, N. K. Pesquisa de marketing: uma orientação aplicada. Tradução Nivaldo M. Jr. e Alfredo A. de Farias. 3. ed. Porto Alegre: Bookman, 200I.

MAMEDE, R. R. Educação em empreendedorismo como fator de desenvolvimento econômico: uma proposta para o município de Campo Grande-MS. In: CONFERENCIA DE INVESTIGACIÓN EN ENTREPRENEURSHIP EN LATINOAMÉRICA, 4., 2005, Santiago de Cali. Anais... Colômbia, 2005. Disponível em: <http://www.oei.es/etp/educacao_empreendedorismo_fator_desemvolvimento_econom co.pdf $>$. Acesso em: I5 abr. 20 Io.

MANKIW, N. G.; ROMER, D.; WEIL, D. N. A contribution to the empirics of economic growth. Quarterly Journal of Economics, v. 107, p. 407-437, 1992. 
MARTELETO, R. M.; SILVA, A. B. Redes e capital social: o enfoque da informação para o desenvolvimento local. Ci. Inf., Brasília, v. 33, n. 3, p. 4I-49, 2004.

MENDENHALL, W.; SINCICH, T. A second course in business statistics: regression analysis. San Francisco: Dellen and Macmillan, I993.

MINCER, J. Schooling, experience, and earnings. Human Behavior \& Social Institutions n. 2. New York: Columbia University Press, I974.

MINNITI, M.; BYGRAVE, W. D.; AUTIO, E. Executive report. Global Entrepreneurship Monitor (GEM). Babson College; London Business School, 2006. Disponível em: <http://www.gemconsortium.org>. Acesso em: I2 mar. 2010.

OCDE. Fostering entrepreneurship. Paris: OECD, I998.

OLIVEIRA, C. A. Uma análise espacial dos impactos do crescimento econômico e da concentração de renda na pobreza dos municípios nordestinos na década de noventa. In: ENCONTRO REGIONAL DE ECONOMIA/NORDESTE, II., 2006, Fortaleza. Fortaleza: BNB, Anpec, 2006. p. 23. Disponível em: <http://www.bnb.gov.br/content/aplicacao/Eventos/forumbnb2006/docs/ uma_analise_especial.pdf>. Acesso em: I2 fev. 20 Io.

OLIVEIRA SILVA, V. H. Crescimento economia e equidade social nos municípios cearenses: uma evidência empírica entre I99I e 2000. In: ENCONTRO DA ECONOMIA DO CEARÁ, 2., 2006, Fortaleza. Anais... Fortaleza: UFC, IPCE, 2006. p. I-I5.

PRITCHETT, L. Where has all the education gone? World Bank Policy Research Working Paper I58I, I996.

REYNOLDS, P. D.; STOREY, D. J.; WESTHEAD, P. Cross national comparison of the variation on the new firm formation rates. Regional Studies, v. 28, n. 4, p. 443-456, Sept. I994.

ROCHA, L. A. Impacto do programa bolsa familia sobre o bem-estar das familias beneficiadas no estado do Ceará. 2008. Dissertação (Mestrado em Economia Rural)-Universidade Federal do Ceará, Fortaleza, 2008.

ROMER, P. Endogenous technological change. The Journal of Political Economy, v. 98, n. 5, p. 7I-IO2, I990.

ROMER, D. Advanced macroeconomics. 2. ed. McGraw-Hill, $200 \mathrm{I}$.

SALA-I-MARTIN, X. Measuring aggregate human (with Casey Mulligan). NBER Working Paper 50I6. Journal of Economic Growth, Feb. I995. Disponível em: <http://www.columbia.edu/ ×s23/ papers/hkmeasm.htm>. Acesso em: 2 fev. 2009.

SCHULTZ, T. O valor econômico da educação. Rio de Janeiro: Zahar, I962.

SCHUMPETER, J. A. Teoria do desenvolvimento econômico. Tradução L. Schlaepfer. Rio de Janeiro: Fundo de Cultura, I96I.

VAN STEL, A.; CARREE, M.; THURIK, R. The effect of entrepreneurial activity on national economic growth. Small Business Economics, v. 24, n. 3, p. 3II-2I, Dec. 2005. 